\title{
INFERENCE OF POLYMER ADSORPTION FROM ELECTRICAL DOUBLE LAYER MEASUREMENTS
}

\author{
J. LYKLEMA \\ Laboratory for Physical and Colloid Chemistry of the Agricultural University, De Dreyen 6, Wageningen, \\ The Netherlands
}

\begin{abstract}
A discussion is given on the use of electrical double layer measurements as a tool for the study of polymer conformations in the adsorbed state. The emphasis is on polymers at solid particles. Combination of data from electrophoresis and direct surface charge measurements leads to information on the polymer segment distribution between trains and loops. The silver iodide-polyvinylalcohol system is chosen as the main example.
\end{abstract}

\section{INTRODUCTION}

More often than not, solid particles, dispersed in aqueous solvents, have a charge on their surface. Together with the equal, but opposite charge in the solution around that particle, it forms the electrical double layer around that particle. Under favourable conditions, information on the composition of this double layer is experimentally accessible.

As a consequence, polymers adsorbed on particles in aqueous solution are as a rule situated in an electrical double layer. In many cases, the properties of this double layer are affected more strongly by the presence of the polymer than that the conformation of the adsorbed macromolecule depends on the presence of the double layer. The study of the influence of polymer adsorption on the double layer properties then offers, in principle, a means to obtain information on the conformation of the adsorbed polymer, having a relatively wide range of validity. This possibility constitutes the theme of this paper. Besides this, in those cases where the adsorbed polymer conformation does depend clearly on the double layer properties, the study of this dependence can reveal interesting information.

\section{SOME BASIC PRINCIPLES}

The usual way to deal with electrical double layers is by treating the solution side as composed of two parts: (1) a non-diffuse molecular condensor or Stern-layer, ${ }^{1}$ sometimes subdivided into an inner and outer Helmholtz layer; ${ }^{2}(2)$ a diffuse or Gouy layer ${ }^{3}$ in which charge and potential obey the Poisson-Boltzmann distribution.

The Stern-layer is the site of specific interactions with the surface, whereas the Gouy-layer is entirely nonspecific. The division is an abstraction of the physical reality of a perhaps more gradual transition from fully specific to fully nonspecific with increasing distance $x$ from the surface. Experience over several decades has shown that this abstraction works satisfactorily.

With polymer adsorption the situation is similar. The actual course $\rho(x)$ of the segment density as a function of the distance from the surface generally depends on such factors as the adsorbed amount $\Gamma_{p}$, the excluded volume, chain flexibility, quality of the solvent etc. ${ }^{4-16}$ The direct influence of the surface is of course strongly felt by the segments in actual contact with it, the so-called train segments. However, this direct influence decays rapidly with $x$. Again, the abstraction can be made that all direct influence of the surface is thought to be condensed in the layer of train segments only, neglecting it in loops and trains. The theory of polymer adsorption is not yet checked completely enough by experiments to state how good this picture is, but it certainly serves well as a first approximation.

More quantitatively, the thickness of the Stern-layer and train segment layer are of similar magnitude, say of the order of $0.5 \mathrm{~mm}$. Neither the thickness of the diffuse double layer part nor that of the layer of loop and tail segments are sharply defined because of the gradual decay with distance. For a flat, unperturbed diffuse layer, the thickness is usually given as the reciprocal Debye length, $\kappa^{-1}$. It is the distance over which the potential $\psi$ decays to the $\mathrm{e}^{-1}$ part of its value $\psi_{d}$ at the outer Helmholtz plane (OHP), at least if $\psi_{d}$ is low. This thickness depends on the concentrations and valencies of the ions present in the system. In $10^{-3} \mathrm{M} \mathrm{KCl}$ it is $10 \mathrm{~nm}$, in $1 \mathrm{M} \mathrm{KCl}$ it is only $0.1 \mathrm{~nm}$. Assigned effective polymer layer thicknesses $\Delta$ depend of course also on a picture of the distribution $\rho(x)$. Moreover, different techniques (viscosimetry, ellipsometry) may measure different effective thicknesses. Actual values, reported both in theory and experiment, vary widely. They depend on the nature of the polymer, its molecular weight $\mathrm{M}$ and the quality of the solvent, but are usually of the order of one to several tens of $\mathrm{nm}$. Thus, we arrive at the following important conclusion: Any modification of the Stern-layer is only attributable to adsorption of train segments, whereas modification of the Gouy-layer is essentially attributable to the presence of loops and tails.

In concentrated electrolyte solutions, the double layer is essentially non-diffuse, hence in this case only the effect of train segments is "seen". In several examples reported in literature, this distinction was not adequately recognized, leading to incorrect conclusions.

The situation is illustrated in Fig. 1, presenting the adsorption of an uncharged polymer in a double layer. The figure is schematic and has no pretentions with respect to the reality of the distribution over trains, loops and tails. The potential drop over the Stern-layer is linear if it is a charge-free layer of constant dielectric permittivity; it is probably also linear if it contains a discrete charge because of multiple imaging. ${ }^{17}$ In the drawing, $\psi_{d}$ is about a third of the surface potential $\psi_{0}$ This is the situation for e.g. AgI-particles at pAg 12 and $10^{-3} \mathrm{M} \mathrm{KNO}_{3}$. In $10^{-1} \mathrm{M} \mathrm{KNO}_{3}, \psi_{d}$ is about $10 \%$ of $\psi_{0}$. For the interpretation it is very important to realize that $\psi_{d}$ is always much smaller than $\psi_{0}$ and dependent on both the salt concentration and the amount of polymer adsorbed in trains. 


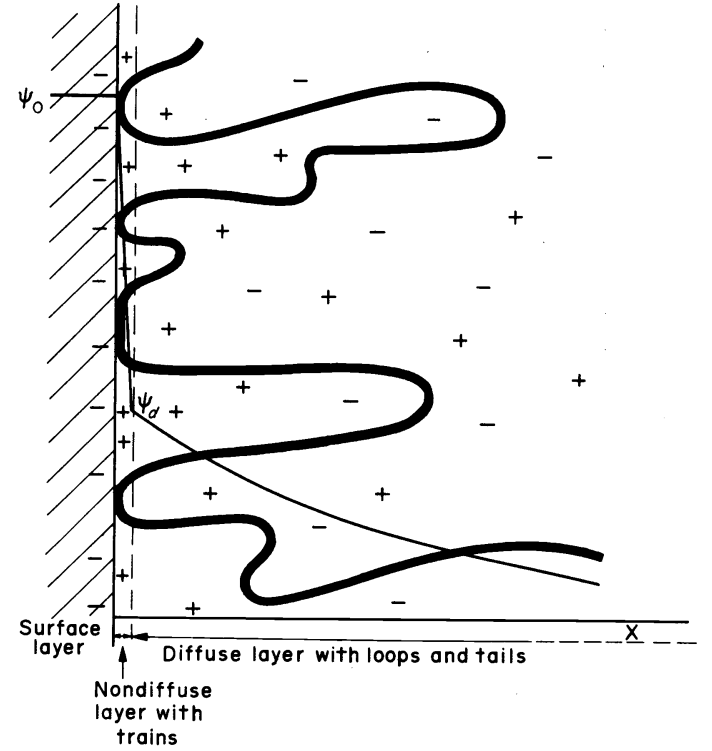

Fig. 1. Schematic picture of a polymer, adsorbed at a charged interface. The course of the potential $\psi$ is indicated.

\section{EXPERIMENTAL PROCEDURES}

As is usually the case, interfacial electrochemistry experiments in the presence of adsorbed polymers can be divided in two groups:

(1) techniques, based on a feature specific to a certain group or surface and hence giving detailed answers in special cases only, and

(2) more generally applicable procedures, yielding as a rule more general, averaged information.

Examples of the first group are the polarographic reduction of $-\mathrm{S}-\mathrm{S}-$ bonds in proteins, adsorbed on mercury ${ }^{18-19}$ or the inhibition of the catalytic decomposition of hydrogen peroxide by colloidal gold due to adsorbed gelatine. ${ }^{20}$ In this paper we are more interested in general methods, especially if they are applicable to disperse systems. The most important of them are the study of the influence of polymer adsorption on (1) the double layer capacitance or on the surface charge, and (2) on the electrophoretic mobility (or on other electrokinetic quantities), all of this in combination with direct measurement of the adsorbed amount $\Gamma_{p}$.

Capacitance measurements are virtually restricted to mercury and hence they have only indirect significance for the study of disperse systems. Another typical feature is that they are usually measured on growing mercury droplets, which brings the rate of spreading and/or the rate of supply by diffusion of polymers in the picture. Although this feature is interesting as such, the various rate-depending factors demonstrate themselves in a quite different fashion with solid surfaces. This is another reason why measurements on mercury are not characteristic of dispersed systems. On the other hand, capacitance measurements on mercury can often be done with relatively great precision, and for their interpretation recourse can be made on an abundance of good data in the presence of adsorbed low molecular weight substances. ${ }^{2,21-27}$ The mercury work with biopolymers has been reviewed by Miller c.s. ${ }^{18,19}$ Generally, the literature on double layer studies on mercury with polymers, ${ }^{28-35}$ polyelectrolytes and biopolymers $s^{18,19,36-46}$ is not abundant and on metals other than mercury ${ }^{20,47}$ practically absent. A difficulty inherent to the mercury system is that as a rule
$\Gamma_{p}$ is not independently measurable. In connection with this, sometimes no proper discrimination is made between differing rate determining processes. For example, the rate of supply of entire molecules by diffusion ${ }^{18,19,32}$ is not directly related to the rate of equilibration of the capacitance in concentrated solutions because the latter only reflects the segments adsorbed in trains. At fixed $\Gamma_{p}$, the distribution over trains, loops and tails can change slowly and irreversibly over hours due to reconformation processes with long relaxation times.

In disperse systems, the available interfacial area is usually sufficiently large to enable direct measurements of $\Gamma_{p}$. However, with these systems no direct capacitance measurements are possible. On mercury, the differential capacitance $C=\mathrm{d} \sigma_{0} / \mathrm{d} \psi_{0}$ can conveniently be measured because of its polarizable interface. However, for dispersed particles the surface is usually reversible in the electrochemical sense, implying that the surface charge $\sigma_{0}$ follows from adsorption of potential-determining ions, e.g. for silver iodide

$$
\sigma_{0}=F\left(\Gamma_{\mathrm{Ag}^{+}}-\Gamma_{\mathrm{I}^{-}}\right)
$$

and for oxides

$$
\sigma_{0}=F\left(\Gamma_{\mathrm{H}^{+}}-\Gamma_{\mathrm{OH}^{-}}\right)
$$

where $F$ is the Faraday and $\Gamma_{i}$ the surface excess of $i$. Equations (1) and (2) are the basis for the direct analytical determination of the surface charge. Potentiometric titration ${ }^{49,50}$ yields $\sigma_{0}(\mathrm{pAg})$ or $\sigma_{0}(\mathrm{pH})$ curves respectively. If the potential-determining ions are constituent ions of the solid (as is the case for $\mathrm{Ag}^{+}$and $\mathrm{I}^{-}$ions with $\mathrm{AgI}$ but not for $\mathrm{H}^{+}$and $\mathrm{OH}^{-}$ions with oxides), the variation of their concentration can be converted to $\mathrm{d} \psi_{0}$ according to Nernst's law:

$$
\mathrm{d} \psi_{0}=-\mathrm{RT} \operatorname{dpAg} / F
$$

allowing the establishment of the differential capacitance, if desired. For oxides, this conversion is not possible without detailed information on the composition of the double layer. ${ }^{50}$

For clays, the charge is due to isomorphic substitution and is invariant if the time scale of the experiment is small as compared with the time scale of ion diffusion in the solid. ${ }^{52}$ Systems with strong acid or basic groups (such as polystyrene sulfonic acid lattices) are also systems of constant surface charge. In the framework of our theme, the invariability of this charge can be a drawback since it implies that one variable is lost.

Surface charges are characteristically measured in high concentrations of indifferent electrolytes, rendering the double layer essentially non-diffuse. Consequently, interpretation in the presence of adsorbed polymers involves only train segments.

Electrokinetic techniques, taking electrophoresis as the representative, are typically applied in dilute electrolytes. The information they produce is complementary to that of surface charge measurements, e.g. because the outward shift of the slipping layer due to polymer adsorption is one of the factors affecting the electrokinetic or $\zeta$ potential. Under favourable conditions, the variation of $\zeta$ can be related to an effective layer thickness $\Delta$. Besides this, shifts of the isoelectric point (i.e.p) can be measured and compared with shifts of the point of zero charge (p.z.c.), derived from potentiometric titration. These quantities again reflect train segment adsorption. 
It follows from this discussion that, because of the complementary nature of $\sigma_{0}$ - and $\zeta$-measurements, their combination with $\Gamma_{p}$ measurements produces the most complete information regarding the distribution of segments over trains, loops and tails.

Quantitative elaboration of this combination of methods requires the solution of several experimental difficulties. Moreover, a number of theoretical pitfalls must be avoided. We shall now discuss its application to real systems, taking polyvinyl alcohol (PVA) adsorbed on AgI as the example. Since PVA is uncharged, the total charge is just the charge on the solid surface and is measurable (1). Another advantage of this system is that double layer studies with related low-molecular weight substances are available. ${ }^{53-55}$

\section{SURFACE CHARGE MEASUREMENTS. \\ THE SILVER IODIDE-PVA SYSTEM}

Figure 2 gives a set of $\sigma_{0}(\mathrm{pAg})$ curves of $\mathrm{AgI}$ in $10^{-1} \mathrm{M}$ $\mathrm{KNO}_{3}$ at various concentrations of PVA. The general features of this set of curves are similar to those in the presence of low-molecular weight adsorbates ${ }^{33,55}$ viz, upon adsorption the slopes of the curves decrease, the point of zero charge moves to the left and all curves pass through a common intersection point $\left(\sigma_{\mathrm{om}}, \mathrm{pAg}\right)$. However, before analyzing them, it is necessary to consider some experimental complications.

The first point is that if polymers adsorb on suspended solids, there is a fair chance that not the entire surface becomes covered because the polymer does not penetrate into narrow pores and/or because clusters of particles are collectively covered by an external sheath. In that case, the double layer properties on the unavailable part of the area remain unaltered. In other words: polymer molecules then "see" a lower adsorbing area than potentialdetermining ions or, for that matter, the effective specific surface areas for polymer and potential-determining ions would be different. It will generally depend on the natures

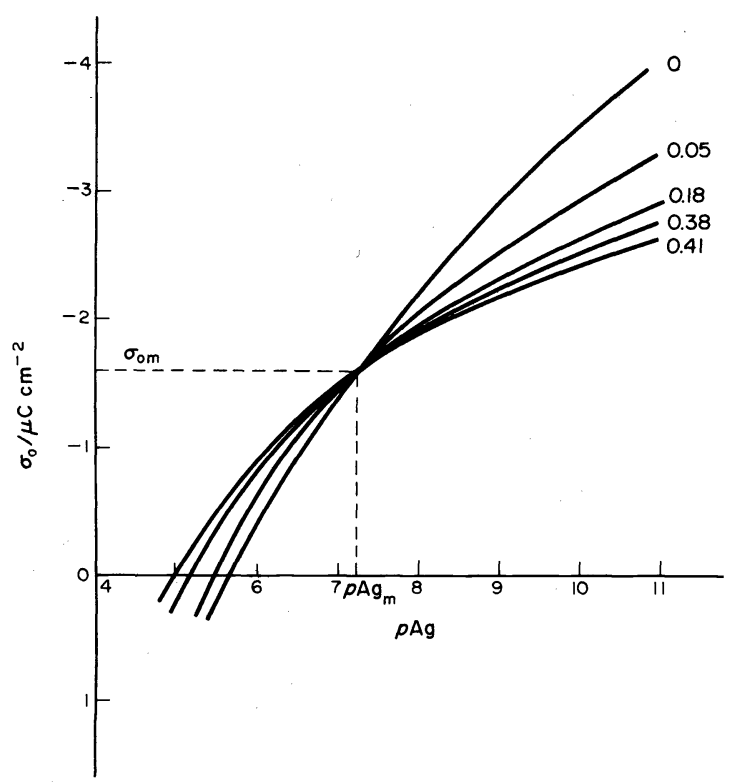

Fig. 2. Surface charge on dispersed silver iodide in the presence of adsorbed polyvinylalcohol $(M=106,500$, containing $2 \%$ acetate groups). Electrolyte: $10^{-1} \mathrm{M} \mathrm{KNO}, T=20.0^{\circ} \mathrm{C}$. The adsorbed amounts $\Gamma_{p}$ in $\mathrm{mg} \mathrm{m}^{-2}$ of the polymer are indicated. of the solid and the polymers, and perhaps also on the procedure of mixing and the suspension concentration, what values must be assigned to these effective areas. No general rules can be given. In our case, an extensive study by Koopal ${ }^{56}$ (involving comparative adsorption measurements of potential-determining ions, a cyanine dye, methylene blue and PVA on various AgI-suspensions and sols) has e.g. shown that on suspended AgI less PVA can be accommodated than on AgI-sols under otherwise similar conditions. With respect to the quantitative interpretation of the curves of Fig. 2 the consequence is that, except at zero polymer concentration, the measured charge is actually a "mixed" charge, consisting say of a fraction $f$ of the unmodified charge $\sigma_{0}(\theta=0)$ and of $(1-f)$ of the modified charge $\sigma_{0}(\theta)$, if $\theta$ stands for the degree of occupancy of the surface with train segments. By virtue of the mutual independence of the covered and uncovered parts of the area at fixed pAg it is justifiable to split up $\sigma_{0}$ according to a linear combination principle

$$
\sigma_{0}(\text { apparent })=f \sigma_{0}(\theta)+(1-f) \sigma_{0}(\theta=0) .
$$

As $\sigma_{0}(\theta=0)$ is well-known, ${ }^{49}$ this allows the evaluation of the sought quantity $\sigma_{0}(\theta)$. It follows from the discussion that considerable errors can be made if the unavailability of part of the area is not accounted for.

The second point is that $\sigma_{0}(\theta)$ is an averaged property. If the adsorption of the polymer on the available area is inhomogeneous, $\sigma_{0}(\theta)$ gives of this inhomogeneous layer only a "smeared-out" bit of information. This restriction is inherent in our method, but it is also inherent in many other techniques, although not always properly recognized.

The third point is the issue of reversibility. Strictly speaking, the various methods of interfacial thermodynamics that are currently used to analyse double layer data in the presence of adsorbed low molecular weight substance ${ }^{2,21-27}$ apply only if the adsorption is a reversible process. However, this statement requires further specification. Adsorption can be irreversible with respect to variation in the polymer concentration but reversible with respect to changes of $\mathrm{pAg}$, in which case $\mathrm{d} \theta / \mathrm{dpAg}$ may be subjected to thermodynamic analysis. Furthermore, the issue of reversibility applies only to that part of the adsorbed material that is actually "seen" in the measurement under consideration. For example, in $10^{-1} \mathrm{M}$ $\mathrm{KNO}_{3}$ solutions only the extent of reversibility of train segment adsorption counts. In the majority of the cases studied, polymer adsorption as a function of concentration is an irreversible process and the PVA-silver iodide system is no exception. Neither are the $\sigma_{0}(\mathrm{pAg})$-curves entirely reversible. A slight lack of reversibility is also sometimes observed in the presence of adsorbing low-molecular weight adsorbates ${ }^{53,55}$ and is perhaps due to unexplained changes in the solid itself. However, with polymers there is a superimposed small but recurrent hysteresis, probably reflecting the non-instantaneous adaptation of the train segment adsorption to alterations of $\mathrm{pAg}$. These effects are not large, usually of the order of $0.1 \mu \mathrm{C} \mathrm{cm}^{-2}$. The curves of Fig. 2 have all been measured at decreasing pAg. Hysteresis-like phenomena or at least phenomena reflecting processes with long relaxation times have also been observed on mercury. For example, Miller and Grahame ${ }^{28}$ found longer (and for the duration of the experiment incomplete) equilibration at lower temperatures for polymethacrylic acid, and Flemming $^{41}$ reported a kind of hysteresis in $C\left(\psi_{0}\right)$ curves for 
DNA, which the author attributed to association phenomena.

The occurrence of a common intersection point for all

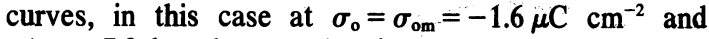
$\mathrm{pAg}_{\mathrm{m}}=7.3$, has also some bearing on this. Hitherto, such a point has been found with all types of PVA on AgI. ${ }^{57,58}$ It is very sharp if located by short titrations around $\sigma_{\mathrm{om}}$ and variable polymer concentration. With low-molecular weight adsorbates ${ }^{53,55}$ it occurs also, as it does on mercury (see e.g. Ref. 59). In the latter cases it has a very definite physical meaning, viz. it is then the surface charge where the surface excess $\Gamma_{a}^{(w)}$ of the organic substance $a$ with respect to water is a maximum as a function of $\sigma_{0}$. For AgI

$$
\Gamma_{a}^{(w)}(\mathrm{pAg})-\Gamma_{a}^{(w)}(\mathrm{pAg})=-\frac{\mathrm{RT}}{F} \int_{\mathrm{pAgm}}^{\mathrm{pAg}}\left(\frac{\delta \sigma_{\mathrm{o}}}{\delta \mu_{a}}\right)_{\mathrm{T}} \mathrm{dpAg}
$$

where the RHS is $<0$ for $\mathrm{pAg} \neq \mathrm{pAg}_{\mathrm{m}}$. Equation (5) follows from the Gibbs equation, i.e. from reversible thermodynamics. The very occurrence of the intersection point with polymers then automatically leads to the conclusion that train segment adsorption is also reversible as a function of $\mathrm{pAg}$, provided the changes in $\mathrm{pAg}$ are not extremely large, this sorption being a maximum at $\sigma_{\mathrm{o}}=\sigma_{\mathrm{om}}^{*} \dagger$ This reversibility over short pAg-stretches and the near-reversibility over long ones has been used by Koopal and Lyklema ${ }^{57}$ to derive for the Stern-layer

$$
\sigma_{0}(\theta)=(1-\theta) \sigma_{0}(\theta=0)+\theta \sigma_{0}(\theta=1)
$$

an equation that is presumably applicable even if the total adsorption is not reversible as a function of concentration. In passing, it can be noted that the sharpness of the intersection point implies that the segment adsorption maximum (as a function of $\sigma_{\mathrm{o}}$ ) itself is independent of $\theta$, which in turn may be taken as an argument for weak or absent lateral interaction or, in other words, an argument supporting linear combinations like (6).

Equation (6) states that the surface charge of a polymer-covered AgI-particle may be written as a linear combination of the charges on a covered and on an uncovered particle, with fractions $\theta$ and $(1-\theta)$ respectively. From (6) the fraction $\theta$ of the surface, covered by train segments, can be deduced if $\sigma_{0}(\theta=1)$ is known. This quantity is not directly experimentally accessible because with polymers it is likely that there remain voids present in the Stern-layer, a feature that has sometimes been overlooked in the corresponding work with mercury. ${ }^{32}$ However, in the AgI-PVA case it is possible to estimate $\sigma_{\mathrm{o}}(\theta=1)$ by using the electrochemical similarity of a fully covered PVA-layer with a hypothetical layer, covered for $50 \%$ with $\mathrm{CH}_{3}$-groups (as in butanol) and for $50 \%$ with $\mathrm{CHOH}$-groups (as in ethylene glycol). The quantitative arguments for this are derived from a comparison of the slopes of the $\sigma_{\mathrm{o}}(\mathrm{pAg})$ curves, the point of zero charge shifts (or isoelectric point shifts) and the values of $\sigma_{\text {om }}$ for the double layer on silver iodide in the presence of butanol, ethylene glycol and PVA.$^{53,55-58}$. It was found that the maximum value of $\theta$ amounts to 0.85 , implying the

†The occurrence of a maximum of $\theta$ as a function of $\sigma_{\mathrm{o}}$ or $\psi_{\mathrm{o}}$ has also been found with macromolecules on mercury, e.g. by Yoshida et al. $^{33}$

$¥$ The choice of the cation has been made on experimental grounds and is without any consequence in our analysis. presence of $15 \%$ voids in the Stern-layer even upon complete coverage. Similar figures have been reported by other investigators with other systems. For example, Miller and Grahame, working with polymethacrylic acid on mercury, found a maximum coverage by train segments of about $80 \%$. $^{28}$

The final conclusion is that the degree of coverage $\theta$ of the Stern-layer by train segments can be evaluated, but this evaluation consists of several steps and requires some prior knowledge. Hence, the procedure is not likely to become a routine method. With respect to our theme, the study of adsorbed polymers by double layer methods, the main significance of the developed method is that it offers an alternative to non-electrochemical methods, and that it can be used in situ. Beyond this scope, the importance of the method for the study of double layers as such can be mentioned. In this context, the electrochemical interpretation of the value of $\sigma_{\text {om }}$, the shift of the point of zero charge and the slopes of the $\sigma_{\mathrm{o}}(\mathrm{pAg})$ curves (except for a constant identical to the double layer capacitance) are important points of discussion.

\section{ELECTROPHORESIS. THE AgI-PVA SYSTEM}

Electrokinetic phenomena, if studied in dilute electrolytes, can yield information not only on the effective thickness $\Delta$ of the adsorbed polymer layer, but also on the adsorption of train segments.

Electrophoretic mobilities in $10^{-3} \mathrm{M} \mathrm{HNO}_{3} \ddagger$ of silver iodide sols, covered with the same PVA as used in Fig. 2, are collected in Fig. 3. The following characteristic features are observed:

(1) the isoelectric point moves to the left with increasing $\Gamma_{p}$;

(2) there is a plateau of the negative side with a shallow maximum; the plateau mobility is reduced by polymer adsorption;

(3) the slope $\mathrm{d}(U / X) / \mathrm{dpAg}$ at the isoelectric point decreases with increasing $\Gamma_{p}$.

The shift of the isoelectric point may be identified with the shift of the point of zero charge (Fig. 2), because at this point specific adsorption is negligible. The isoelectric shift $\Delta$ i.e.p is far more accurately measurable than $\Delta$ p.z.c. Moreover, it is perhaps better amenable to analysis because on sols complete coverage is more likely than on suspensions. The shifts reflect the replacement of water dipoles at the AgI-solution interface by $-\mathrm{CH}_{2}-\mathrm{CHOH}-$ segments. It is characteristic that the first adsorbed molecules produce a relatively strong shift, indicating that they are to a large extent adsorbed in trains. At higher $\Gamma_{p}$, the isoelectric point becomes less sensitive to $\Gamma_{p}$ because, upon progressive adsorption, later adsorbing polymer accumulates itself predominantly in loops and tails which do not affect the location of the isoelectric point. In other words, $\delta^{2}\left|\Delta \mathrm{pAg} \mathrm{g}^{\mathrm{o}}\right| / \delta \Gamma_{p}{ }^{2}<0$. One of the curves in Fig. 4 illustrates this.

It is tempting to interpret the reduction of the plateau mobility with increasing $\Gamma_{p}$ as the qualitative consequence of the outward movement of the slipping plane. However, it is not recommendable to use this decrease for the quantitative establishment of the effective layer thickness $\Delta$ because of the following prohibiting problems:

(1) Because of the difficulty to account properly for retardation, the conversion of mobilities into $\zeta$-potentials is not really feasible in the plateau region ${ }^{60}$ and virtually impossible if the particles are not spherical.

(2) Accepting that reliable values of $\zeta$ have been 


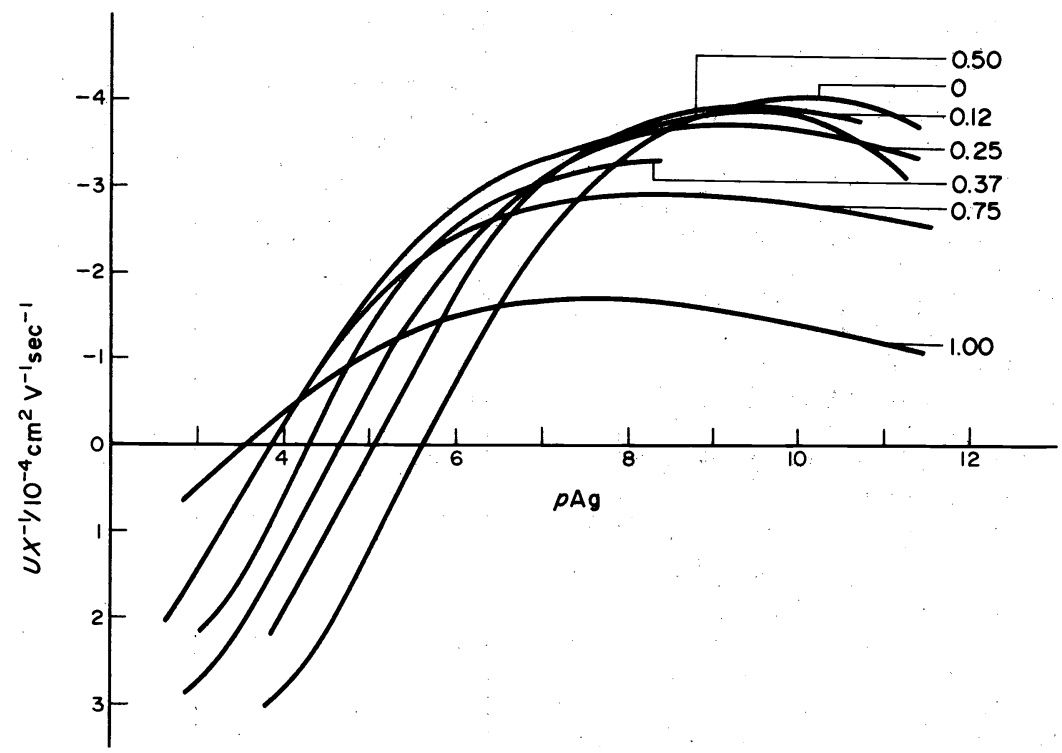

Fig. 3. Electrophoretic mobilities of AgI-sols in the presence of adsorbed polyvinylalcohol $(M=106,500$, containing $2 \%$ acetate groups). Electrolyte: $10^{-3} \mathrm{M} \mathrm{HNO}_{3} . T=20.0^{\circ} \mathrm{C}$. The adsorbed amounts $\Gamma_{p}$ in $\mathrm{mg} \mathrm{m}^{-2}$ of the polymer are indicated.

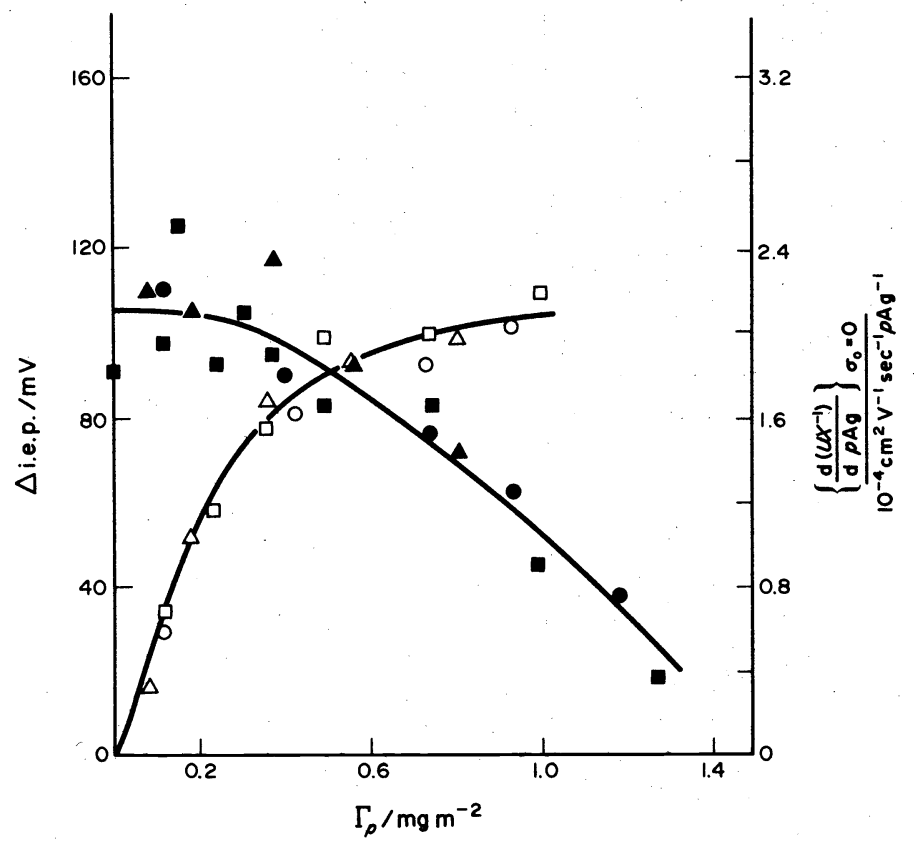

Fig. 4. Shift of the isoelectric point (left) and of the slope $\mathrm{d}(U / X) / \mathrm{dpAg}$ in the isoelectric point ( point of zero charge) for AgI-sols in the presence of adsorbed polyvinylalcohol, containing $2 \%$ acetate groups. Molecular weights: $\Delta, \Delta$ 15,$500 ; 0,060,000 ; \square, \square 106,500$. Electrolyte: $10^{-3} \mathrm{MHO}_{3} ; \mathrm{T}=20.0^{\circ} \mathrm{C}$.

established, the calculation of $\Delta$ requires both the value of $\psi_{d}$ (Fig. 1$)$ and the course $\psi(x)$ between the OHP and the slipping plane. These bits of information are not generally available. There are no independent procedures leading to unambiguous determination of $\psi_{d}$. This quantity can be obtained from titration data only in favourable cases, such as the case of no specific adsorption, whereas under plateau conditions specific adsorption is nonzero and dependent on the amount of polymer adsorbed in trains. The behaviour of $\psi(x)$ has been studied theoretically by Brooks. ${ }^{61}$

Brooks bases his theory on a collection of experi- ments ${ }^{62}$ in which the adsorption of uncharged dextrans on various surfaces (erythrocytes, silica, polystyrene lattices) often led to an increase of $\zeta$. He then proceeds to show that, due to the presence of adsorbed polymers, the diffuse part of the double layer can expand. Actually, instead of the Boltzmann distribution of counterions,i.e.

$$
n_{i}(x)=n_{i}(\infty) \exp \left(-\frac{z_{i} \mathrm{e} \psi(x)}{k T}\right)
$$

he obtains, using the Flory-Huggins treatment of concen- 
trated polymer solutions,

$$
n_{i}(x)=n_{i}(\infty) \exp \left(-\frac{z_{i} \mathrm{e} \psi(x)}{k T}\right) \cdot \exp \{-\beta(x)\}
$$

with

$$
\left.\beta(x)=\left\{\phi_{p}(x)-\phi_{p}(\infty)\right\} 1-\chi_{s p}+\chi_{i p}-\chi_{s i}\right\}
$$

where $\phi_{p}$ stands for the volume fraction of polymer and $\chi$ for the Flory-Huggins interaction parameter, the subscripts $i, s$ and $p$ referring to the counterion, solvent and polymer respectively. The correction factor $\beta(x)$ is a product of which the first factor accounts for the purely geometrically excluded volume, whereas the second accounts for the effect of non-ideality on the distribution. The first factor is always positive and less than unity; it leads to an expansion of the diffuse layer. The second factor is probably also positive, its magnitude depending on the natures of polymer, ion and solvent. If the modified Boltzmann eqn (8) is combined with the Poisson equation, the potential distribution $\psi(x)$ over the polymer layer can be evaluated. For low potentials it was found that, due to the presence of polymer chains, the potentials rise all over the polymer sheath, including the boundary between the rigid and free draining liquid. In the quantitative derivation, Brooks represents $\phi_{p}(x)$ by a step-function, but the qualitative conclusion remains valid for an exponential decay $\rho(x)$ because of general electrostatic considerations.

The conclusion is that there are virtually two counteracting trends: (a) the increasing distance betwen the plane of shear and OHP upon polymer adsorption leads to a reduction of $\zeta$, and (b) the alteration of the potentialand charge distribution by polymer leads to an increase of $\zeta$. It is permissible to invoke trend (a) as a means to evaluate $\Delta$ only if trend (b) can be shown to be insignificant or if it is quantitatively accounted for. However, the quantitative solution in turn requires, besides accurate parameter values, also some insight into the distribution $\rho(x)$ which is just the quantity we want to establish.

With respect to the application to actual systems some more comments need to be made. In the first place, we recall that with uncharged polymers on charged particles experimentally both increases ${ }^{62}$ and decreases ${ }^{63}$ (see also Fig. 3) are found. Closer inspection of Fig. 3 reveals that not far from the isoelectric point at constant pAg the electrophoretic mobility rises with increasing $\Gamma_{p}$, which is just the opposite of what happens in the platform region. The explanation is of course that, due to the shift of the isoelectric point, $\psi_{\mathrm{o}}$ and $\psi_{d}$ become larger. This feature is not covered by Brooks' theory, at least not explicitly. A second point is that it has been shown with low-molecular weight adsorbates that $\psi_{d}$ may increase due to the displacement of counterions from the Stern-layer by organic molecules. ${ }^{54}$ The possible occurrence of this feature must also be anticipated with polymer trains. All these complications render the application of this theory to the evaluation of $\Delta$ virtually impossible. This, of course, does not detract from the intrinsic value of the theory. Experimental tests with well-defined systems remain desirable.

After concluding that the reduction of $\zeta$ in the platform region is not easily related to $\Delta$, we propose instead of this to use the slope of the mobility as a function of $\mathrm{pAg}$ at the isoelectric point or in a narrow range around it. The underlying idea is that this slope decreases with increasing $\Delta$. Since the analysis is done at $\sigma_{\mathrm{o}}=0$, no difficulties arise with specific adsorption and both relaxation corrections or Brooks-type corrections are small or absent. Experiments in the presence of polymer offer no special difficulty. On the contrary, they may be easier because of steric stabilization of the dispersed particles.

For a flat double layer in an (1-1) electrolyte, the following equation can be derived: ${ }^{57}$

$$
\left(\frac{\mathrm{d} \zeta}{\mathrm{dpAg}}\right)_{\sigma_{0} \rightarrow 0}=58.16 \frac{\operatorname{sech}^{2}\left(\mathrm{e} \psi_{d} / 4 k T\right)}{\operatorname{sech}^{2}(\mathrm{e} \zeta / 4 k T)} \cdot \frac{C}{C_{d}} \exp (-\kappa \Delta)
$$

and for a spherical double layer, using the Debye-Hückel approximation,

$$
\left(\frac{\mathrm{d} \zeta}{\mathrm{dpAg}}\right)_{\sigma_{0} \rightarrow 0}=58.16 \frac{C_{a} \exp \cdot(-\kappa \Delta)}{C_{d}(a+\Delta)} .
$$

In these equations, $C$ is the total double layer capacitance which can be obtained from titration and $C_{d}$ the capacitance of the diffuse part. The latter can be derived from theory, thereby noting that $C_{d}$ depends on curvature. As both the potentials and the electrolyte concentrations are low, it is fully justified to neglect specific adsorption. Then,

$$
\frac{1}{C}=\frac{1}{C_{m}}+\frac{1}{C_{d}}
$$

where $C_{m}=\epsilon_{s} \epsilon_{0} / d$ is the capacitance of the (charge-free) molecular condensor if $\epsilon_{s}$ is its relative dielectric constant, $\mathrm{d}$ its thickness and $\epsilon_{\mathrm{o}}=8.854 \times 10^{-2} \mathrm{C}^{2} \mathrm{~N}^{-1} \mathrm{~m}^{-2}$. Consequently, for the factor $C / C_{d}$ may also be substituted

$$
C / C_{d}=\left\{1+\left(C_{d} / C_{m}\right)\right\}^{-1} .
$$

Usually $C_{m}$ is of the order of $20 \mu \mathrm{F} \mathrm{cm}^{-2}$. For a flat double layer $C_{d}=5.7 \mu \mathrm{F} \mathrm{cm}^{-2}$, hence putting $C / C_{d}$ equal to unity involves an error of $20 \%$. For a spherical particle with radius $33 \mathrm{~nm}$ these figures are $9.5 \mu F \mathrm{~cm}^{-2}$ and $\sim 50 \%$ respectively, indicating that as a rule the distinction between $C$ and $C_{d}$ must be maintained. Finally, it is noted that $\psi_{d}$ in (10) follows directly from $\sigma_{\mathrm{o}}$ which can be established by titration. Ultimately, only $\Delta$ remains in the equations as an unknown.

In many cases no titration data are available. It may be noted that even then the quotient $C / C_{d}$ can be derived from electrophoresis, but only in the absence of polymer. It follows both from (10) and (11) that for $\Delta \rightarrow 0 \psi_{d} \rightarrow \zeta$

$$
\left(\frac{\mathrm{d} \zeta}{\mathrm{dpAg}}\right)_{\substack{\sigma_{0} \rightarrow 0 \\ \Delta \rightarrow 0}}=58.16 \frac{C}{C_{d}}
$$

Using the value for $C / C_{d}$ thus obtained in the presence of adsorbed polymer also is, though not quantitatively correct, a better approximation than putting this quotient equal to unity.

Values of the slopes $(\mathrm{d} \zeta / \mathrm{dpAg})_{\sigma_{0 \rightarrow 0}}$ for PVA on silver iodide are plotted as a function of $\Gamma_{p}$ in Fig. 4. The trend of this curve is just the opposite from that of the shift in the isoelectric point. This is obviously due to the fact that at low $\Gamma_{p}$ most of the adsorbed polymer is accommodated in trains, whereas at high $\Gamma_{p}$ any further increase in $\Gamma_{p}$ takes place progressively in loops and tails.

The question remains what kind of thickness is thus 
measured. This problem is not yet solved, the solution would require a detailed knowledge of the drainage pattern through adsorbed polymer layers. Perhaps it is not too farfetched to presume ${ }^{58}$ that at the slipping plane $\rho(x)$ has the same value as $\rho(r)$ has in a Gaussian coil in solution with $r=r_{h}=\xi r_{g}$ where $r_{h}=$ hydrodynamic radius, $r_{g}=$ radius of gyration. ${ }^{64}$ The theoretical value of $\xi$ is 0.665 , but in practice sometimes other values are given. $^{58,65}$

In view of the relatively easy techniques and interpretation the combination of electrophoresis and short titrations around $\sigma_{0}=0$ appears to be a method that may gain routine character, the more so since graphs of the type of Fig. 4 contain already all required basic information.

\section{RESULTS FOR PVA ON SILVER IODIDE}

Results in terms of $\theta\left(\Gamma_{p}\right)$ and $\Delta\left(\Gamma_{p}\right)$ curves for PVA on silver iodide are collected in Fig. 5 . The outcome is very much as intuitively expected: at low $\Gamma_{p}$ adsorption proceeds mainly in trains. However, with increasing $\Gamma_{p}$ the polymer adsorbed in excess is progressively allocated to loops and tails.

A striking feature is that within experimental accuracy the same $\theta\left(\Gamma_{p}\right)$ and $\Delta\left(\Gamma_{p}\right)$ curves are found for samples of PVA, differing between each other with respect to acetate content (2-12\%) and molecular weights $\left(1.4 \times 10^{3}-1.3 \times\right.$ $\left.10^{4}\right)$. The conclusion is that the distribution over trains and loops or tails is a function of the adsorbed amount only, although of course the adsorption isotherms $\Gamma_{p}\left(c_{p}\right)$ are different. If our finding could be generalized, it would considerably simplify the treatment of colloidal phenomena involving polymer-covered particles.

Before finishing this section, it can be noted that the results of Fig. 5 do not cover any statement with respect to the type of segment distribution $\rho(x)$ (exponential, Gaussian, step-function etc.), the physical interpretation

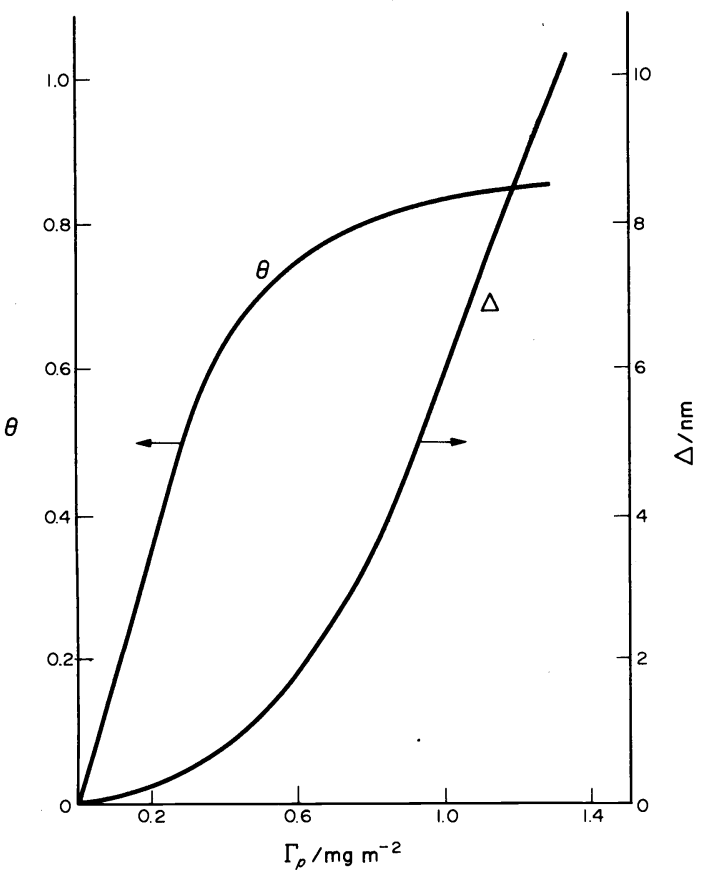

Fig. 5. Characterization of PVA, adsorbed on silver iodides by methods of interfacial electrochemistry. Given are the degree of occupancy $\theta$ of train segments in the Sternlayer and the electrophoretic thickness $\Delta$. of the quantity $\Delta$ being dependent on the nature of the distribution.

\section{OTHER SYSTEMS}

As far as we are aware, no other suspended material has been sufficiently thoroughly studied to apply our interfacial electrochemistry techniques.

With oxides, the technique leading to $\sigma_{0}(\mathrm{pH})$ curves does not work. In our laboratory, Van der Linde has found that neither $\sigma_{0}$ nor the point of zero charge of hematite $\left(\alpha-\mathrm{Fe}_{2} \mathrm{O}_{3}\right)$ suspensions was affected by adsorption of PVA. These results were supported by Kavanagh $e t$ al. for PVA on gibbsite $\left(\mathrm{Al}_{2} \mathrm{O}_{3}\right)^{63}$ We suggest that this is due to the fact that on oxides the surface charge is not located on the outer surface, but penetrates into the interior of the solid. ${ }^{66}$ This part is not "seen" by polymer. Thus the insensitivity of oxide double layers to polymers underscores the "porous double layer" notion, although it excludes the application of our technique.

The procedure elaborated for the PVA-silver iodide system is expected to be generally applicable with uncharged polymers and non-porous solids of known potential-determining mechanism. In several instances analogies with other systems have been detected. It requires considerable modification if the macromolecule is charged. For systems of constant charge (clays, polystyrene latex) the application is limited since the influence of the adsorbed polymer on $\sigma_{o}$ is now no longer a variable.

The results of our analysis may be considered as a step in the treatment of the properties of polymer-covered disperse systems, notably their colloidal stability.

\section{CONCLUSION}

The discussion on the potentialities of methods of interfacial electrochemistry to obtain information on the distribution over trains and loops or tails of adsorbed polymer segments has led to the conclusion that the most recommendable technique consists of short potentiometric titrations and electrophoresis measurements around the point of zero charge. The procedure has been worked out for polyvinylalcohol on silver iodide. In this case the distribution of segments over trains and loops is a function of the adsorbed amount only.

The results of this electrochemical work can be compared with results from non-electrochemical sources. Moreover, they contribute to the understanding of the composition of electrical double layers in general.

Acknowledgement-The author thanks Mr. L. K. Koopal for his help in the construction of this paper and for his consent to use his experimental data.

\section{REFERENCES}

1O. Stern, Z. Elektrochem. 30, 508 (1924).

${ }^{2}$ D. C. Grahame, Chem. Rev. 41, 441 (1947).

${ }^{3}$ G. Gouy, J. Phys. 9(4), 457 (1910); Ann. Phys. 7(9), 129 (1917).

${ }^{4}$ A. Silberberg, J. Phys. Chem. 66, 1872, 1884 (1962).

${ }^{5}$ E. A. Di Marzio, J. Chem. Phys. 42, 2101 (1965)

${ }^{6}$ C. A. J. Hoeve, E. A. Di Marzio and P. Peyser, J. Chem. Phys. 42, 2558 (1965).

${ }^{7}$ E. A. Di Marzio and F. M. McCrackin, J. Chem. Phys. 43, 539 (1965).

${ }^{8}$ R. J. Rubin, J. Chem. Phys. 43, 2392 (1965).

${ }^{9} \mathrm{C}$. A. J. Hoeve, J. Chem. Phys. 43, 3007 (1965).

${ }^{10}$ M. L. Huggins, Makromolek. Chem. 87, 119 (1965).

${ }^{11}$ A. Silberberg, J. Chem. Phys. 46, 1105 (1967).

${ }^{12}$ A. Silberberg, J. Chem. Phys. 48, 2835 (1968). 
${ }^{13}$ C. A. J. Hoeve, J. Polymer Sci. (C) 34, 1 (1971).

${ }^{14}$ A. Silberberg, Faraday Discuss. Chem. Soc. $59(1975)$.

${ }^{15}$ F. Th. Hesselink, J. Phys. Chem. 73, 3488 (1969); 75, 65 (1971).

${ }^{16}$ F. Th. Hesselink, J. Colloid Interfac. Sci. 50, 606 (1975).

${ }^{17}$ D. C. Grahame, Z. Elektrochem. 62, 261 (1957).

${ }^{18}$ I. R. Miller and D. Bach, in Surface and Colloid Sci., (Edited by

E. Matijevič), Vol. 6, p. 185. Wiley, New York (1973).

${ }^{19}$ I. R. Miller in Progress in Surface and Membrane Science (Edited by J. F. Danielli, M. D. Rosenberg and D. A. Cadenhead), Vol. 4, p. 299. Academic Press, New York (1971).

${ }^{20} \mathrm{M}$. Caselli and A. Traini, J. Electroanal. Chem. 50, 387 (1974).

${ }^{21} \mathrm{~J}$. A. V. Butler, Electrical Phenomena at Interfaces. Methuen, London (1951).

${ }^{22}$ R. Parsons, Ann. Repts. Chem. Soc. 6180 (1964).

${ }^{23}$ B. E. Conway, Theory and Principles of Electrode Processes. Ronald Press, New York (1965).

${ }^{24}$ M. A. V. Devanathan and B. V. K. S. R. A. Tilak, Chem. Revs. 65, 635 (1965).

${ }^{25}$ E. Gileadi, Electrosorption. Plenum Press, New York (1967).

${ }^{26} \mathrm{M}$. J. Sparnaay, The Electrical Double Layer. Pergamon Press, Oxford (1972).

${ }^{27} \mathrm{R}$. Payne, Double layer at the mercury-solution interface, in Progress in Surface and Membrane Science (Edited by J. F. Danielli, M. D. Rosenberg and D. A. Cadenhead), Vol. 6, p. 51. Academic Press, New York (1973).

${ }^{28}$ I. R. Miller and D. C. Grahame, J. Am. Chem. Soc. 78, 3577 (1956).

${ }^{29}$ W. Wójciak and E. Dutkiewicz, Roczn. Chem. 38, 271 (1964).

${ }^{30}$ J. B. Craig, R. Bain and P. Meares, Kolloid-Z.Z. Polym. 221, (1967).

${ }^{31} \mathrm{H}$. Jehring and E. Horn, Abh. Deut. Akad. Wissensch. Berlin 10, 295 (1968).

${ }^{32}$ T. Yoshida, T. Ohsaka and S. Tanaka, Bull. Chem. Soc. Japan 45, 326 (1972).

${ }^{33}$ T. Yoshida, T. Ohsaka and H. Yamamoto, Bull. Chem. Soc Japan 45, 3236 (1972).

${ }^{34}$ T. Yoshida, T. Ohsaka and M. Suzuki, Bull. Chem. Soc. Japan 45, 3245 (1972)

${ }^{35}$ L. Marszall, Colloid and Polymer Sci. 252, 335 (1974).

${ }^{36} \mathrm{~J}$. Flemming, Biopolym. 6, 2697 (1968).

${ }^{37}$ V. Brabec, Biopolym. 11, 2577 (1972).

${ }^{38}$ V. Brabec, J. Electroanal. Chem. 50, 235 (1974).

${ }^{39}$ N. E. Enukidze and G. A. Tedoradze, Soobshch. Akad. Nauk. Gruz. SSR 68, 709 (1972); 69, 449 (1973).
${ }^{40} \mathrm{~J}$. Flemming, J. Electroanal. Chem. 47, 137 (1973).

${ }^{41} J$. Flemming, Biopolym. 12, 1975 (1973).

${ }^{42}$ B. Janik and R. G. Sommer, Biopolym. 12, 2803 (1973).

${ }^{43}$ P. Valenta and P. Grahmann, J. Electroanal. Chem. 49, 41 (1974).

${ }^{4} \mathrm{P}$. Valenta and H. W. Nürnberg, J. Electroanal. Chem. 49, 55 (1974).

${ }^{45} \mathrm{P}$. Valenta, H. W. Nürnberg and P. Klahre, Bioelectrochem. and Bioenerg. 1, 487 (1974).

${ }^{46}$ P. Valenta and H. W. Nürnberg, Biophys. Struct. Mech. 1, 17 (1974).

${ }^{47}$ T. Ohsaka, Y. Abe and T. Yoshida, Bull. Chem. Soc. Japan 46, 1324 (1973)

${ }^{48}$ O. Pavlovič and I. R. Miller, J. Polymer Sci. (C) 34, 181 (1971).

49. Lyklema and J. Th. G. Overbeek, J. Colloid Sci. 16, 595(1961).

${ }^{50}$ Th. F. Tadros and J. Lyklema, J. Electroanal. Chem. 17, 267 (1968).

${ }^{51}$ S. Levine and A. L. Smith, Discuss. Faraday Soc. 52, 290 (1972).

${ }^{32} \mathrm{H}$. van Olphen, An Introduction to Clay Colloid Chemistry. Interscience, New York (1963).

${ }^{53}$ B. H. Bijsterbosch and J. Lyklema, J. Colloid Sci. 20, 665 (1965).

${ }^{54} \mathrm{~B}$. Vincent, B. H. Bijsterbosch and J. Lyklema, J. Colloid Interfac. Sci. 37, 171 (1971).

${ }^{55}$ J. N. de Wit and J. Lyklema, J. Electroanal. Chem. 41, 259 (1973).

${ }^{56}$ L. K. Koopal, Thesis, Landbouwhogeschool Wageningen, Netherlands, in preparation.

${ }^{57}$ L. K. Koopal and J. Lyklema, Faraday Discuss. Chem. Soc. 59, 230 (1975).

${ }^{58}$ G. J. Fleer, L. K. Koopal and J. Lyklema, Kolloid-Z.Z. Polym. 250, 689 (1972)

${ }^{59}$ E. Blomgren, J. O'M. Bockris and C. Jesch, J. Phys. Chem. 65, 2000 (1961)

${ }^{60}$ P. H. Wiersema, A. Loeb and J. Th. Overbeek, J. Colloid Interfac. Sci. 22, 78 (1966).

${ }^{61}$ D. E. Brooks, J. Colloid Interfac. Sci. 43, 687 (1973)

${ }^{62}$ D. E. Brooks and G. V. F. Seaman, J. Colloid Interfac. Sci. 43 670 (1973).

${ }^{63}$ B. V. Kavanagh, A. M. Posner and J. P. Quirk, Faraday Discuss. Chem. Soc. 59 (1975).

${ }^{64} \mathrm{C}$. Tanford, Physical Chemistry of Macromolecules. Chap. 22. Wiley, New York (1961).

${ }^{65}$ M. J. Garvey, Th. F. Tadros and B. Vincent, J. Colloid Interfac. Sci. 49, 57 (1974).

${ }^{66}$ J. Lyklema, J. Electroanal. Chem. 18, 341 (1968). 